ARTIGO DE REVISÃO

THALES VIANNA COUTINHO

MARIANA RODRIGUES GONÇALVES DIAS
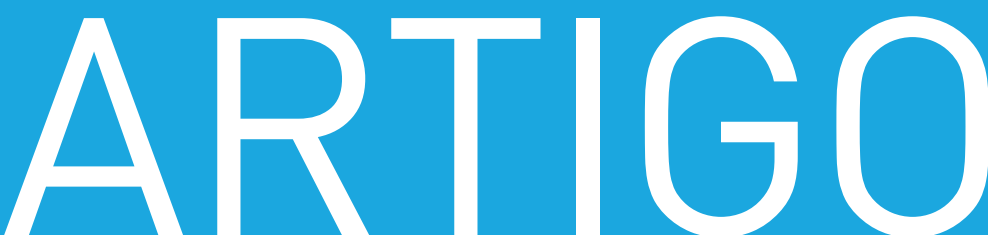

\title{
CONTAMINACÃO MENTAL NO TRANSTORNO OBSESSIVO-ĆOMPULSIVO: REVISÃO DA LITERATURA E ORIENTAÇÕES PARA A PESQUISA E A CLINIICA DURANTE A PANDEMIA DA COVID-19
}

\section{MENTAL CONTAMINATION IN OBSESSIVE-COMPULSIVE DISORDER: LITERATURE REVIEW AND GUIDELINES FOR RESEARCH AND CLINICAL PRACTICE DURING THE COVID-19 PANDEMIC}

\section{Resumo}

É possível se contaminar através do pensamento? Objetivamente não; subjetivamente, porém, não se pode dizer o mesmo. O construto "contaminação mental" ainda é muito pouco estudado e, principalmente, pouco difundido na literatura em língua portuguesa. Sendo assim, o objetivo deste artigo foi revisar os estudos que relacionaram a contaminação mental ao transtorno obsessivo-compulsivo (TOC) ao longo dos últimos 5 anos. A busca foi realizada através das bases de dados PubMed, MEDLINE e PsycINFO, e após a aplicação dos critérios de inclusão, seis estudos foram revisados integralmente. Com base na análise das amostras utilizadas, dos instrumentos adotados, dos resultados encontrados e das limitações informadas pelos autores de cada estudo, concluímos que: 1) poucos estudos contemplaram tanto uma população clínica como uma não clínica; 2) o Vancouver Obsessional Compulsive Inventory-Mental Contamination foi o instrumento mais utilizado para mensurar a contaminação mental; 3) a contaminação mental tem relação com outros fenômenos que também fazem parte do TOC (como a propensão ao nojo); e 4) os estudos apresentam, comumente, limitações relacionadas à amostra. Ao final, apresentamos os passos necessários para incentivar a pesquisa científica brasileira acerca da contaminação mental, bem como estratégias que os profissionais da saúde mental podem adotar para orientar pacientes com TOC, durante o período da pandemia, visando reduzir a suscetibilidade à contaminação mental.

Palavras-chave: Transtorno obsessivo-compulsivo, contaminação mental, COVID-19.

\section{Abstract}

Is it possible to become contaminated through thought? Not objectively, but subjectively yes. The construct referred to as "mental contamination" is still poorly investigated and rarely mentioned in Portuguese-language scientific literature. Therefore, the aim of this article was to review studies that correlated mental contamination to obsessive-compulsive disorder (OCD) over the past 5 years. The search was performed on the PubMed, MEDLINE and PsycINFO databases; after applying the inclusion criteria, six studies were fully reviewed. Based on the analysis of samples, instruments adopted, results found and limitations reported by the authors of each study, we conclude that: 1) few studies have examined both clinical and non-clinical samples; 2) the Vancouver Obsessional Compulsive Inventory-Mental Contamination was the instrument most commonly used to measure mental contamination; 3) mental contamination is related to other phenomena that are also present in OCD (e.g., 


\section{COVID-19}

THALES VIANNA COUTINHOํㅡ, MARIANA RODRIGUES GONC̣ALVES DIAS²

1 Doutorando, Programa de Medicina Molecular, Faculdade de Medicina, Universidade Federal de Minas Gerais (UFMG), Belo Horizonte, MG. ${ }^{2}$ Mestranda, Programa de Ciências da Saúde: Saúde da Criança e do Adolescente, Faculdade de Medicina, UFMG, Belo Horizonte, MG.

disgust propensity); and 4) the studies commonly have sample limitations. Towards the end of the text, we present the necessary steps to encourage Brazilian scientific research on mental contamination and also suggest how mental health professionals can guide patients with OCD during the pandemic, to reduce susceptibility to mental contamination.

Keywords: Obsessive-compulsive disorder, mental contamination, COVID-19.

\section{INTRODUÇÃO}

A contaminação mental pode ser definida como a percepção subjetiva de que se está contaminado, mesmo não tendo contato algum com pessoa ou objeto que pudesse fisicamente provocar contaminação1. Por exemplo, o indivíduo pode se sentir sujo ao se lembrar de uma situação do passado em que se sujou e sentir a necessidade de se higienizar mesmo assim. A contaminação mental produz, então, a sensação de sujeira interna, que leva o indivíduo a desejar lavar as mãos².

Ainda que a contaminação mental possa ocorrer em situações normais, ela se torna uma ameaça à saúde mental quando assume um caráter incontrolável, constante, que provoca estresse e gera prejuízos funcionais ${ }^{3}$.

Sabe-se que existem alguns fatores psicológicos que tornam os indivíduos mais vulneráveis a manifestar a contaminação mental, sendo eles: elevada sensibilidade ao nojo (intensidade com que se sente enojado diante de alguma situação nojenta); medo da contaminação (superestimativa da probabilidade de se contaminar, seguida pelo desejo de higienização); e sensibilidade à ansiedade (receio de experienciar reações relacionadas à ansiedade) $)^{4}$.

Quando procuramos compreender o papel da contaminação mental nas condições psiquiátricas, o transtorno obsessivo-compulsivo (TOC) é o principal exemplo. O TOC está presente em aproximadamente $1,7 \%$ da população ${ }^{5}$ e pode ser caracterizado pela presença de obsessões (pensamentos recorrentes e tidos como intrusivos e irracionais) muito ansiógenas, que quase sempre impelem o paciente a realizar alguma compulsão (comportamento repetitivo, ritualístico e tido como irresistível) que, momentaneamente, alivia a ansiedade ${ }^{6}$.
Pacientes com TOC, especialmente aqueles com obsessões relativas à contaminação, vivenciam frequentemente a contaminação mental que, inclusive, está relacionada à severidade do quadro ${ }^{7}$. Entretanto, é fundamental ressaltar que a contaminação mental também está presente naqueles pacientes com obsessão por pensamentos envolvendo a possibilidade de causar dano a si mesmo ou a terceiros, conteúdos proibidos e/ou de desejo por simetria, demonstrando que esse fenômeno faz parte do TOC de uma maneira bastante generalizada ${ }^{8}$.

Ainda que o receio da contaminação seja muito frequente entre os pacientes com TOC, há algumas diferenças que merecem ser destacadas entre a contaminação por contato e a contaminação mental. Na primeira, a sensação de contaminação é decorrente do contato físico com algo, a higienização é eficiente para amenizar a ansiedade, e a fonte é considerada mais tangível; enquanto na segunda, a contaminação ocorre na ausência de um contato direto, os rituais de higienização são menos eficientes e a fonte da contaminação é percebida como intangível?.

Apesar de a maioria dos estudos sobre contaminação mental envolvendo população clínica ter sido realizada com pacientes com TOC, alguns estudos consideram que a contaminação mental pode ser entendida como um construto transdiagnóstico, presente em diferentes quadros ${ }^{10,11}$, como o transtorno do estresse póstraumático ${ }^{12,13}$ e até mesmo em mulheres que sobrevivem à violência sexual ${ }^{14}$.

É necessário ressaltar que, apesar da relevância para a compreensão do TOC, a contaminação mental não é mencionada na $10^{a}$ edição da Classificação Internacional de Doenças (CID-10), nem na $5^{a}$ edição do Manual Diagnóstico e Estatístico dos Transtornos Mentais (DSM-5). Além disso, até o momento da escrita deste artigo, não foram encontrados trabalhos sobre o tema publicados em língua portuguesa. Esses dados levam a crer que, possivelmente, uma parcela significativa dos profissionais da saúde mental brasileiros desconhece a existência da contaminação mental. Revela-se, então, um cenário problemático, especialmente porque a psicoeducação com relação à contaminação mental deve ser parte integrante do protocolo de tratamento bem-sucedido para o $\mathrm{TOC}^{15}$. 


\section{ARTIGO DE REVISÃO}

THALES VIANNA COUTINHO MARIANA RODRIGUES GONÇALVES DIAS
Em virtude dos impasses supracitados, resolvemos elaborar a presente revisão da literatura. Foram contemplados trabalhos publicados ao longo dos últimos 5 anos, a fim de atualizar o conhecimento dos profissionais da saúde mental, bem como incentivar que pesquisas nacionais sejam desenvolvidas sobre essa temática. Ainda, devido à recente pandemia da COVID-19, propomos, ao final, orientações aos clínicos para que possam oferecer uma assistência mais completa aos pacientes com TOC.

\section{MÉTOdo}

Realizou-se uma busca por artigos nas bases de dados PubMed, MEDLINE e PsycINFO, utilizando as seguintes palavras-chave: mental contamination e obsessivecompulsive disorder.

Adotou-se, como critério de inclusão, que seriam consideradoselegíveis paraestarevisãoartigospublicados durante os últimos 5 anos e que contemplavam amostra clínica com TOC. Artigos de revisão, metanálise ou que não fossem escritos em língua portuguesa ou inglesa foram excluídos.
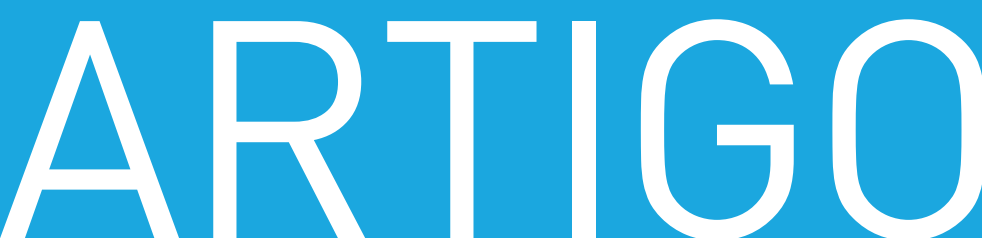

A busca inicial revelou oito artigos. Após a leitura independente do resumo, seis artigos foram considerados elegíveis, por estarem de acordo com os critérios acima descritos, para a leitura completa e incorporação dos dados à revisão que se segue.

\section{Resultados}

A Tabela 1 sintetiza os dados extraídos dos seis artigos que compuseram esta revisão. Com relação à coluna Resultados, ressaltamos que incluímos apenas aqueles que dizem respeito à contaminação mental em si. Isso porque alguns estudos, na etapa inicial, realizaram o processo de validação de instrumentos, mas que foge ao escopo desta revisão. Com relação à coluna Limitações, sintetizamos aquelas listadas pelos próprios autores dos estudos, não sendo realizado julgamento por parte dos autores desta revisão. A tabela foi construída seguindo a ordem cronológica das publicações.

Com relação às amostras, apenas dois dos seis estudos incluíram tanto amostra clínica quanto não clínica, permitindo a comparação entre os grupos.

Tabela 1 - Dados dos artigos que compuseram a revisão

\begin{tabular}{|c|c|c|c|c|c|}
\hline Estudo & $\begin{array}{l}\text { Amostra } \\
\text { clínica }\end{array}$ & $\begin{array}{l}\text { Amostra } \\
\text { não clínica }\end{array}$ & $\begin{array}{l}\text { Instrumentos } \\
\text { utilizados }\end{array}$ & Resultados & Limitações \\
\hline \multirow{7}{*}{$\begin{array}{l}\text { Coughtrey } \\
\text { et al. }{ }^{15}\end{array}$} & \multirow[t]{7}{*}{15} & \multirow[t]{7}{*}{0} & \multirow{7}{*}{$\begin{array}{l}\text { The Mental } \\
\text { Contamination Imagery } \\
\text { Interview }\end{array}$} & \multirow{2}{*}{$\begin{array}{l}\text { Imagens mentais podem engatilhar a } \\
\text { contaminação mental. }\end{array}$} & Amostra pequena; \\
\hline & & & & & Todos os participantes estavam \\
\hline & & & & \multirow{2}{*}{$\begin{array}{l}\text { Dos participantes que experienciaram } \\
\text { as imagens mentais que induziam à } \\
\text { contaminação mental, metade relatou que } \\
\text { eram na forma de fotografias, enquanto } \\
\text { que a outra metade informou que eram na } \\
\text { forma de pequenos videoclipes. }\end{array}$} & $\begin{array}{l}\text { atualmente em tratamento para } \\
\text { TOC; }\end{array}$ \\
\hline & & & & & $\begin{array}{l}\text { Viés do experimentador na } \\
\text { interpretação das respostas dos } \\
\text { participantes. }\end{array}$ \\
\hline & & & & $\begin{array}{l}\text { Metade dos participantes que } \\
\text { manifestaram a imagem mental chegou a } \\
\text { lavar as próprias mãos como uma estratégia } \\
\text { para aliviar a sensação de sujeira provocada } \\
\text { pela imagem mental. }\end{array}$ & \\
\hline & & & & $\begin{array}{l}\text { A maioria dos participantes que } \\
\text { experienciaram as imagens mentais relatou } \\
\text { que as visualizavam na perspectiva de } \\
\text { primeira pessoa. }\end{array}$ & \\
\hline & & & & $\begin{array}{l}\text { Alguns participantes conseguiram utilizar } \\
\text { outras imagens mentais (por exemplo, de } \\
\text { memórias alegres) para diminuir o impacto } \\
\text { que a imagem de contaminação provocava. }\end{array}$ & \\
\hline
\end{tabular}




\section{COVID-19}

THALES VIANNA COUTINHOํㅣㄹ, MARIANA RODRIGUES GONC̣ALVS DIAS²

1 Doutorando, Programa de Medicina Molecular, Faculdade de Medicina, Universidade Federal de Minas Gerais (UFMG), Belo Horizonte, MG. ${ }^{2}$ Mestranda, Programa de Ciências da Saúde: Saúde da Criança e do Adolescente, Faculdade de Medicina, UFMG, Belo Horizonte, MG.

Tabela 1 - Continua

\begin{tabular}{|c|c|c|c|c|c|}
\hline Estudo & $\begin{array}{l}\text { Amostra } \\
\text { clínica }\end{array}$ & $\begin{array}{l}\text { Amostra } \\
\text { não clínica }\end{array}$ & $\begin{array}{l}\text { Instrumentos } \\
\text { utilizados }\end{array}$ & Resultados & Limitações \\
\hline \multirow[t]{7}{*}{$\begin{array}{l}\text { Melli et } \\
\text { al. }^{16}\end{array}$} & $\begin{array}{l}120 \\
\text { pacientes } \\
\text { com TOC }\end{array}$ & \multirow[t]{7}{*}{541} & $\begin{array}{l}\text { Vancouver Obsessional } \\
\text { Compulsive Inventory - } \\
\text { Mental Contamination }\end{array}$ & \multirow{7}{*}{$\begin{array}{l}\text { A VOCI-MC conseguiu discriminar os } \\
\text { pacientes com TOC que apresentaram um } \\
\text { escore significativamente maior daqueles } \\
\text { com outros transtornos de ansiedade. }\end{array}$} & \multirow[t]{7}{*}{$\begin{array}{l}\text { Amostra clínica que dificultou a } \\
\text { análise estatística. }\end{array}$} \\
\hline & \multirow{6}{*}{$\begin{array}{l}31 \text { com } \\
\text { diagnóstico } \\
\text { de outros } \\
\text { transtornos } \\
\text { de } \\
\text { ansiedade }\end{array}$} & & Scale (VOCl-MC) & & \\
\hline & & & $\begin{array}{l}\text { Obsessive-Compulsive } \\
\text { Inventory-Revised }\end{array}$ & & \\
\hline & & & $\begin{array}{l}\text { Beck Depression } \\
\text { Inventory-II }\end{array}$ & & \\
\hline & & & Beck Anxiety Inventory & & \\
\hline & & & $\begin{array}{l}\text { Social Interaction and } \\
\text { Anxiety Scale }\end{array}$ & & \\
\hline & & & $\begin{array}{l}\text { Dimensional Obsessive- } \\
\text { Compulsive Scale }\end{array}$ & & \\
\hline \multirow[t]{7}{*}{$\begin{array}{l}\text { Zysk et } \\
\text { al. }{ }^{17}\end{array}$} & \multirow[t]{7}{*}{140} & \multirow[t]{7}{*}{760} & $\begin{array}{l}\text { Morphing Fear } \\
\text { Questionnaire (MFQ) }\end{array}$ & \multirow{7}{*}{$\begin{array}{l}\text { O escore elevado na MFQ apresentou } \\
\text { correlação mais forte com o escore de } \\
\text { OCI-R e VOCI-MC que com os de BAI } \\
\text { e BDI-II, sugerindo que o medo de ter a } \\
\text { própria identidade transformada ao ter } \\
\text { contato com alguém com características } \\
\text { indesejáveis e/ou reprováveis pode ser } \\
\text { um sintoma relacionado à contaminação } \\
\text { mental. }\end{array}$} & \multirow{7}{*}{$\begin{array}{l}\text { A amostra clínica foi definida } \\
\text { por autorrelato, não sendo } \\
\text { baseada em um diagnóstico } \\
\text { formalmente realizado. }\end{array}$} \\
\hline & & & VOCI-MC & & \\
\hline & & & $\begin{array}{l}\text { Obsessional } \\
\text { Compulsive Inventory - } \\
\text { Short Version (OCl-R) }\end{array}$ & & \\
\hline & & & $\begin{array}{l}\text { Thought-Action Fusion } \\
\text { Scale }\end{array}$ & & \\
\hline & & & Magical Ideation Scale & & \\
\hline & & & $\begin{array}{l}\text { Beck Anxiety Inventory } \\
\text { (BAI) }\end{array}$ & & \\
\hline & & & $\begin{array}{l}\text { Beck Depression } \\
\text { Inventory - II (BDI-II) }\end{array}$ & & \\
\hline \multirow{9}{*}{$\begin{array}{l}\text { Melli et } \\
\text { al. }^{16}\end{array}$} & \multirow[t]{9}{*}{169} & \multirow[t]{9}{*}{0} & Contamination Fear & \multirow{7}{*}{$\begin{array}{l}\text { Correlação significativa entre contaminação } \\
\text { mental e propensão ao nojo. } \\
\text { A contaminação mental age como fator } \\
\text { mediador da relação entre a propensão } \\
\text { ao nojo e o medo de contaminação por } \\
\text { contato baseado no nojo de evitação. }\end{array}$} & Não foi incluído grupo controle; \\
\hline & & & Core Dimensıons scale & & \multirow{4}{*}{$\begin{array}{l}\text { Os participantes foram } \\
\text { selecionados com base no } \\
\text { autorrelato; } \\
\text { Não é possível definir relação } \\
\text { de causa e efeito; }\end{array}$} \\
\hline & & & VOCI-MC & & \\
\hline & & & Disgust Propensity & & \\
\hline & & & Questionnaire & & \\
\hline & & & $\begin{array}{l}\text { Obsessive-Compulsive } \\
\text { Inventory-Revised }\end{array}$ & & \multirow{2}{*}{$\begin{array}{l}\text { Todos os dados derivam de } \\
\text { medidas de autorrelato; }\end{array}$} \\
\hline & & & Beck Depression & & \\
\hline & & & Inventory-II & & \multirow{2}{*}{$\begin{array}{l}\text { A contaminação mental e } \\
\text { propensão ao nojo são apenas } \\
\text { duas das muitas variáveis que } \\
\text { provavelmente influenciam o } \\
\text { medo de contaminação. Seria } \\
\text { interessante concluir outras } \\
\text { variáveis. }\end{array}$} \\
\hline & & & Beck Anxiety Inventory & & \\
\hline
\end{tabular}




\section{ARTIGO DE REVISÃO}

THALES VIANNA COUTINHO

MARIANA RODRIGUES GONÇALVES DIAS

Tabela 1 - Continua

\begin{tabular}{|c|c|c|c|c|c|}
\hline Estudo & $\begin{array}{l}\text { Amostra } \\
\text { clínica }\end{array}$ & $\begin{array}{l}\text { Amostra } \\
\text { não clínica }\end{array}$ & $\begin{array}{l}\text { Instrumentos } \\
\text { utilizados }\end{array}$ & Resultados & Limitações \\
\hline \multirow[t]{8}{*}{ Poli et al. ${ }^{18}$} & Estudo 1:0 & Estudo 1: & Three Domains of & \multirow{3}{*}{$\begin{array}{l}\text { Apenas um fator da escala dos três } \\
\text { domínios do nojo, o patogênico, teve } \\
\text { correlação significativa com o escore de } \\
\text { limpeza da escala de TOC, mais associado à } \\
\text { contaminação por contato. }\end{array}$} & Desenho transversal; \\
\hline & \multirow{7}{*}{$\begin{array}{l}\text { Estudo } \\
2: 103\end{array}$} & \multirow{7}{*}{ Estudo 2:0 } & & & Ausência de grupo controle; \\
\hline & & & $\begin{array}{l}\text { Disgust Propensity } \\
\text { Questionnaire }\end{array}$ & & $\begin{array}{l}\text { Critérios questionáveis na } \\
\text { seleção dos participantes; }\end{array}$ \\
\hline & & & $\begin{array}{l}\text { Guilt Sensitivity } \\
\text { Questionnaire }\end{array}$ & \multirow{4}{*}{$\begin{array}{l}\text { Apenas um fator da escala dos três } \\
\text { domínios do nojo, o sexual, teve } \\
\text { correlação significativa com a escala de } \\
\text { contaminação mental. Revelou-se como a } \\
\text { variável preditora do escore na escala de } \\
\text { contaminação mental, independentemente } \\
\text { de sintomas de depressão e ansiedade. }\end{array}$} & $\begin{array}{l}\text { Ausência de instrumentos para } \\
\text { mensurar a emoção raiva; }\end{array}$ \\
\hline & & & $\begin{array}{l}\text { Depression Anxiety } \\
\text { Stress Scales-21 }\end{array}$ & & \multirow{2}{*}{$\begin{array}{l}\text { Metodologia para avaliação } \\
\text { do transtorno obsessivo- } \\
\text { compulsivo; }\end{array}$} \\
\hline & & & $\mathrm{VOCl}-\mathrm{MC}$ & & \\
\hline & & & Beck Anxiety Inventory & & \multirow{2}{*}{$\begin{array}{l}\text { Todos os dados derivam de } \\
\text { medidas de autorrelato; }\end{array}$} \\
\hline & & & $\begin{array}{l}\text { Beck Depression } \\
\text { Inventory-II }\end{array}$ & $\begin{array}{l}\text { O fator moral da escala dos três domínios } \\
\text { do nojo não apresentou correlação nem } \\
\text { com a contaminação mental nem com a } \\
\text { contaminação por contato. }\end{array}$ & \\
\hline \multirow[t]{8}{*}{$\begin{array}{l}\text { Mathes et } \\
\text { al. }{ }^{19}\end{array}$} & \multirow[t]{8}{*}{88} & \multirow[t]{8}{*}{0} & $\begin{array}{l}\text { Mini International } \\
\text { Neuropsychiatric } \\
\text { Interview }\end{array}$ & \multirow{2}{*}{$\begin{array}{l}\text { A contaminação mental faz com que o } \\
\text { medo de contaminação persista mesmo } \\
\text { após remover algum possível contaminante } \\
\text { físico, através da higienização, mantendo o } \\
\text { comportamento compulsivo. }\end{array}$} & \multirow{3}{*}{$\begin{array}{l}\text { Todos os participantes } \\
\text { receberam o mesmo } \\
\text { tratamento, e não está claro se } \\
\text { os efeitos são específicos ao } \\
\text { ERP ou ao tratamento em geral; }\end{array}$} \\
\hline & & & $\mathrm{VOCl}-\mathrm{MC}$ & & \\
\hline & & & $\begin{array}{l}\text { Dimensional Obsessive } \\
\text { Compulsive Scale }\end{array}$ & \multirow{3}{*}{$\begin{array}{l}\text { Um programa envolvendo três sessões de } \\
\text { Exposure and Response Prevention (ERP) } \\
\text { focado nos sintomas de contaminação, seja } \\
\text { por contato ou mental, revelou-se efetivo } \\
\text { para diminuir a intensidade de ambas } \\
\text { as obsessões de contaminação (que são } \\
\text { independentes entre si). }\end{array}$} & \\
\hline & & & $\begin{array}{l}\text { Disgust Propensity and } \\
\text { Sensitivity Scale }\end{array}$ & & \multirow{2}{*}{$\begin{array}{l}\text { A amostra consistiu } \\
\text { principalmente de mulheres } \\
\text { universitárias e caucasianos, } \\
\text { limitando a generalização dos } \\
\text { resultados a outras populações; }\end{array}$} \\
\hline & & & $\begin{array}{l}\text { Contact Contamination } \\
\text { (CC) Task }\end{array}$ & & \\
\hline & & & \multirow[t]{3}{*}{$\begin{array}{l}\text { Mental Contamination } \\
\text { (MC) Task }\end{array}$} & \multirow{2}{*}{$\begin{array}{l}\text { O nível da contaminação mental antes de } \\
\text { o tratamento iniciar prediz o sucesso do } \\
\text { tratamento para reduzir a contaminação } \\
\text { por contato. }\end{array}$} & $\begin{array}{l}\text { Não foram contemplados outros } \\
\text { diagnósticos psiquiátricos; }\end{array}$ \\
\hline & & & & & $\begin{array}{l}\text { Limitação da especificidade do } \\
\text { instrumento VOCl-MC. }\end{array}$ \\
\hline & & & & $\begin{array}{l}\text { A contaminação mental e propensão ao } \\
\text { nojo são construtos significativamente } \\
\text { correlacionados. }\end{array}$ & $\begin{array}{l}\text { Limitações metodológicas que } \\
\text { dificultam a generalização dos } \\
\text { ganhos do tratamento. }\end{array}$ \\
\hline
\end{tabular}

Com relação aos instrumentos utilizados pelos pesquisadores para mensurar a contaminação mental, com exceção de um deles, todos os demais adotaram a Vancouver Obsessional Compulsive Inventory - Mental Contamination Scale (VOCl-MC). Trata-se de um instrumento de autorrelato, com estrutura unifatorial, composto por 20 itens, em que o paciente deve informar o nível de concordância, utilizando uma escala tipo Likert que varia de zero (not at all) a 4 (very much).
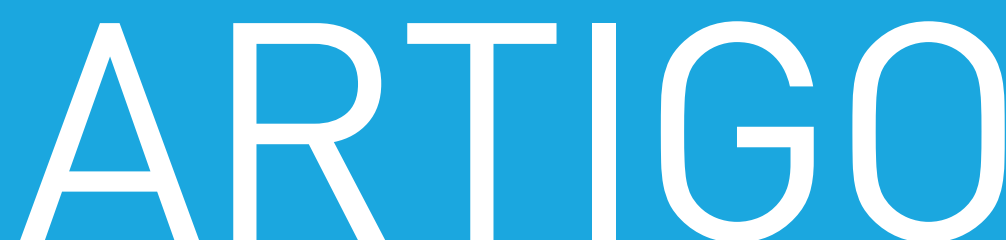

Guilt Sensitivity

Depression Anxiety or sexul, teve ( contaminação mental, independentemente

fator moral da escala dos três domínios contaminação mental nem com a

A contaminação mental faz com que o receberam o mesmo tratamento, e não está claro se os efeitos são específicos ao ERP ou ao tratamento em geral;

A amostra consistiu principalmente de mulheres universitárias e caucasianos, limitando a generalização dos resultados a outras populações:

Não foram contemplados outros Limitação da especificidade do dificultam a generalização dos ganhos do tratamento.
A síntese dos resultados dos estudos permite destacar que, no caso do TOC, a contaminação mental apresenta correlação significativa com a propensão ao nojo ${ }^{16,19}$ e o receio de perder a própria identidade ao interagir com alguém indesejado ${ }^{20}$. Ainda, a contaminação mental também pode ser engatilhada por imagens mentais ${ }^{21}$, bem como fazer perseverar o ritual compulsivo, mesmo após a limpeza das mãos ${ }^{19}$. 


\section{COVID-19}

THALES VIANNA COUTINHO'- MARIANA RODRIGUES GONÇALVES DIAS²

1 Doutorando, Programa de Medicina Molecular, Faculdade de Medicina, Universidade Federal de Minas Gerais (UFMG), Belo Horizonte, MG. ${ }^{2}$ Mestranda, Programa de Ciências da Saúde: Saúde da Criança e do Adolescente, Faculdade de Medicina, UFMG, Belo Horizonte, MG.

Com relação às limitações, percebe-se que problemas envolvendo as amostras foram uma constante, seja no sentido de uma amostra não ser representativa da sociedade como um todo (o que pode prejudicar a generalização dos dados), seja no sentido de amostras reduzidas ou baseadas exclusivamente em autorrelato, não havendo um diagnóstico psiquiátrico formal de TOC.

\section{DISCUSSÃo}

Acontaminação mentaléum construto bastante relevante na avaliação dos casos de TOC e também para o manejo clínico ${ }^{22}$. Entretanto, ainda é um assunto subinvestigado, e no Brasil não há estudos a esse respeito.

Por isso, é de fundamental relevância para a clínica e a pesquisa brasileira não apenas validar a VOCl-MC, por já ser utilizada por praticamente todos os estudos recentes - o que seria uma estratégia mais assertiva do que desenvolver um instrumento novo ${ }^{23}$-, como também convocar uma amostra mais representativa da população, tanto clínica quanto não clínica, evitando vícios que possam prejudicar a generalização dos resultados ${ }^{24}$.

Além disso, é preciso considerar o potencial de intensificação dos rituais compulsivos na contaminação mental, sobretudo em função da demasiada veiculação de palavras e imagens de cunho mórbido decorrentes da atual pandemia da COVID-19. Afinal, conforme já apresentado, as imagens mentais podem engatilhar a contaminação mental, que potencializa o quadro de $\mathrm{TOC}^{21,25}$. Em tese, tais imagens podem ser produzidas por notícias acerca da pandemia, veiculadas em diferentes mídias ${ }^{26}$.

É fato que as notícias da pandemia devem ser transmitidas à população, até em função do seu potencial persuasivo para a adoção de medidas profiláticas necessárias (distanciamento social, uso de máscaras, higienização constante, etc.) ${ }^{27}$. Porém, seria ideal adotar a estratégia proposta pelo professor de psicologia política Michael Petersen, que cunhou o termo ansiedade otimista. Basicamente, o novo conceito diz respeito a uma condição na qual o indivíduo sente a ansiedade em um nível suficiente para torná-lo capaz de entender a severidade da situação, mas também mantém o otimismo necessário para acreditar que as suas ações individuais podem fazer a diferença ${ }^{28}$. Ou seja, se as notícias puderem despertar essa ansiedade otimista no espectador, será muito producente.
Porém, apesar das propostas científicas de protocolo para transmitir as notícias da COVID-1929, sabe-se que os noticiários nem sempre são baseados em tais recomendações. Por isso, é imprescindivel pensar em estratégias para minimizar o efeito potencialmente negativo sobre a saúde mental dos pacientes psiquiátricos, especialmente aqueles com TOC, que acabam sendo constantemente bombardeados com números e imagens que rementem às consequências negativas da contaminação.

Ainda não é possível afirmar que as notícias sobre a COVID-19 possam engatilhar contaminação mental em pacientes com TOC, afinal, até o presente momento, não há estudos acerca disso. Entretanto, uma pesquisa recente concluiu que a exposição a notícias da COVID19 pode potencializar, por exemplo, a xenofobia ${ }^{30}$. Sabe-se, há alguns anos, que a xenofobia tem o nojo como emoção de base ${ }^{31}$, e tal emoção é específica da contaminação ${ }^{32}$. Ou seja, teoricamente, faz sentido tomarmos alguns cuidados quanto ao possível efeito da apreciação de tais notícias. Por isso, recomendamos que os pacientes com TOC sejam orientados no sentido de evitar a superexposição às notícias da pandemia.

Pensando nesse cenário, que pode ser um gatilho para a acentuação de sintomas psiquiátricos, concluise que zelar pela saúde mental é fundamental ${ }^{33}$. Por isso, inclusive, profissionais da saúde estão sendo desencorajados a suspender os seus serviços durante esse período crítico ${ }^{34}$. Afinal, o tratamento clínico aos pacientes psiquiátricos não deve ser interrompido nem mesmo pelo isolamento social, em função da COVID-19, principalmente àqueles cujo quadro pode ser agravado devido ao estresse da pandemia ${ }^{35}$.

Ressaltamos, portanto, que além do risco de adoecimento devido à COVID-19, pacientes psiquiátricos são mais vulneráveis aos aspectos deletérios do estresse relacionado à pandemia, podendo potencializar a ansiedade e agravar o quadro 36 .

Esta revisão apresenta algumas limitações que precisam ser contempladas. A primeira diz respeito ao período adotado para a seleção dos artigos. Em função da janela de 5 anos, não foram revisados outros trabalhos envolvendo o papel da contaminação mental para o TOC. Outra limitação é que não revisamos o impacto da contaminação mental em outras condições 


\section{ARTIGO DE REVISÃO}

THALES VIANNA COUTINHO

MARIANA RODRIGUES GONC̣ALVES DIAS

psiquiátricas, para que fosse possível estabelecer uma melhor comparação com o TOC, o que possibilitaria ressaltar eventuais especificidades. Ainda, como terceira limitação, podemos destacar que devido à ausência de estudos nacionais, não é possível estabelecer se os dados aqui abordados podem ser generalizados à população brasileira. Recomendamos que futuras revisões possam ser conduzidas com uma janela temporal maior e contemplando diferentes condições clínicas afetadas pela contaminação mental.

\section{CONCLUSÕES}

Ressaltamos que, até o momento, esta é a primeira revisão que contemplou a relação entre contaminação mental e TOC. Consideramos o construto como sendo fundamental tanto para a conceitualização clínica como para o manejo dos pacientes com TOC. Ainda, em função da atual pandemia, intervenções mais específicas, visando minimizar a contaminação mental, tais como a redução da exposição às notícias da COVID-19, podem ser necessárias para manter a saúde mental desses pacientes.

Artigo submetido em 15/05/2020, aceito em 16/05/2020. Os autores informam não haver conflitos de interesse associados à publicação deste artigo.

Fontes de financiamento inexistentes.

Correspondência: Thales Vianna Coutinho, Av. Brasil, 673, sala 201, Bairro Santa Efigênia, Belo Horizonte, MG. E-mail: thales.coutinho@hotmail.com

\section{Referências}

1. Rachman S, Radomsky AS, Elliott CM, Zysk E. Mental contamination: the perpetrator effect. J Behav Ther Exp Psychiatry. 2012;43:587-93.

2. Coughtrey AE, Shafran R, Lee M, Rachman SJ. It's the feeling inside my head: a qualitative analysis of mental contamination in obsessive-compulsive disorder. Behav Cogn Psychother. 2012;40:16373.

3. Radomsky AS, Coughtrey A, Shafran R, Rachman S. Abnormal and normal mental contamination. J Obsessive Compuls Relat Disord. 2018;17:46-51.

4. Herba JK, Rachman S. Vulnerability to mental contamination. Behav Res Ther. 2007;45:2804-12.
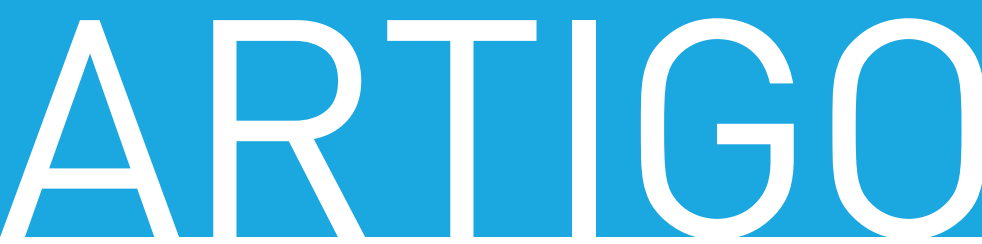

5. Skapinakis P, Politis S, Karampas A, Petrikis $\mathrm{P}$, Mavreas $V$. Prevalence, comorbidity, quality of life and use of services of obsessive-compulsive disorder and subthreshold obsessive-compulsive symptoms in the general adult population of Greece. Int J Psychiatry Clin Pract. 2019;23:215-24.

6. Lilienfeld SO, Pydych AL, Lynn SJ, Latzman RD, Waldman ID. 50 differences that make a difference: a compendium of frequently confused term pairs in psychology. Front Educ. 2017;2:37.

7. Coughtrey AE, Shafran R, Knibbs D, Rachman SJ. Mental contamination in obsessive-compulsive disorder. J Obsessive Compuls Relat Disord. 2012;1:244-50.

8. Jacoby RJ, Blakey SM, Reuman L, Abramowitz JS. Mental contamination obsessions: an examination across the obsessive-compulsive symptom dimensions. J Obsessive Compuls Relat Disord. 2018;17:9-15.

9. Rachman S. Fear of contamination. Behav Res Ther. 2004;42:1227-55.

10. Blakey SM, Jacoby RJ. The polluted mind: understanding mental contamination as a transdiagnostic phenomenon. J Obsessive Compuls Relat Disord. 2018:17:1-2.

11. Coughtrey A, Shafran R, Bennett S, Kothari R, Wade T. Mental contamination: relationship with psychopathology and transdiagnostic processes. J Obsessive Compuls Relat Disord. 2018;17:39-45.

12. Badour CL, Feldner MT, Babson KA, Blumenthal $\mathrm{H}$, Dutton CE. Disgust, mental contamination, and posttraumatic stress: unique relations following sexual versus non-sexual assault. J Anxiety Disord 2013;27:155-62.

13. Brake CA, Jones AC, Wakefield JR, Badour CL. Mental contamination and trauma: understanding posttraumatic stress, risky behaviors, and helpseeking attitudes. J Obsessive Compuls Relat Disord. 2018;17:31-8.

14. Fairbrother N, Rachman S. Feelings of mental pollution subsequent to sexual assault. Behav Res Ther. 2004;42:173-89.

15. Coughtrey AE, Shafran R, Lee M, Rachman S. The treatment of mental contamination: a case series. Cogn Behav Pract. 2013;20:221-31. 


\section{COVID-19}

THALES VIANNA COUTINHO'- MARIANA RODRIGUES GONÇALVES DIAS²

${ }^{1}$ Doutorando, Programa de Medicina Molecular, Faculdade de Medicina, Universidade Federal de Minas Gerais (UFMG), Belo Horizonte, MG. ${ }^{2}$ Mestranda, Programa de Ciências da Saúde: Saúde da Criança e do Adolescente, Faculdade de Medicina, UFMG, Belo Horizonte, MG.

16. Melli G, Bulli F, Carraresi C, Tarantino F, Gelli S, Poli A. The differential relationship between mental contamination and the core dimensions of contact contamination fear. J Anxiety Disord. 2017;45:916.

17. Zysk E, Shafran R, Williams T. The origins of mental contamination. J Obsessive Compuls Relat Disord. 2018:17:3-8.

18. Poli A, Melli G, Radomsky AS. Different disgust domains specifically relate to mental and contact contamination fear in obsessive-compulsive disorder: Evidence from a path analytic model in an Italian clinical sample. Behav Ther. 2019;50:38094.

19. Mathes BM, McDermott KA, Okey SA, Vazquez A, Harvey AM, Cougle JR. Mental contamination in obsessive-compulsive disorder: associations with contamination symptoms and treatment response. Behav Ther. 2019;50:15-24.

20. Shafran R, Zysk E, Williams T. The origins of mental contamination. J Obsessive Compuls Relat Disord. 2018;17:38.

21. Coughtrey AE, Shafran R, Rachman SJ. Imagery in mental contamination. Behav Cogn Psychother. 2015:43:257-69.

22. Rachman S, Coughtrey A, Shafran R, Radomsky A. Oxford guide to the treatment of mental contamination. Oxford: Oxford University; 2014.

23. Borsa JC, Damásio BF, Bandeira DR. Adaptação e validação de instrumentos psicológicos entre culturas: algumas considerações. Paidéia (Ribeirão Preto). 2012;22:423-32.

24. Henrich J, Heine SJ, Norenzayan A. The weirdest people in the world? Behav Brain Sci. 2010;33:6183.

25. Klein JP, Moritz S. On the relevance of mental imagery beyond stress-related psychiatric disorders. Front Psychiatry. 2014;5:77.

26. Krishna A, Cian L, Sokolova T. The power of sensory marketing in advertising. Curr Opin Psychol. 2016;10:142-7.

27. Van Bavel JJ, Baicker K, Boggio PS, Capraro V, Cichocka A, Cikara M, et al. Using social and behavioural science to support COVID-19 pandemic response. Nat Hum Behav. 2020;4:460-71.
28. Petersen $M$. The unpleasant truth is the best protection against coronavirus [Internet]. 2020 Mar 9 [cited 2020 May 20]. pure.au.dk/portal/ files/181464339/The_unpleasant_truth_is_the best_protection_against_coronavirus_Michael_ Bang_Petersen.pdf

29. Finset $\mathrm{A}$, Bosworth $\mathrm{H}$, Butow P, Gulbrandsen $\mathrm{P}$, Hulsman RL, Pieterse $\mathrm{AH}$, et al. Effective health communication-a key factor in fighting the COVID-19 pandemic. Patient Educ Couns. 2020;103:873-6.

30. Sorokowski P, Groyecka A, Kowal M, Sorokowska A, Białek M, Lebuda I, et al. Information about pandemic increases negative attitudes toward foreign groups: a case of COVID-19 outbreak [Internet]. 2020 Mar 31 [cited 2020 May 20]. https://psyarxiv.com/ j23vt/

31. Navarrete CD, Fessler DM. Disease avoidance and ethnocentrism: the effects of disease vulnerability and disgust sensitivity on intergroup attitudes. Evol Hum Behav. 2006;27:270-82.

32. Curtis $\vee$, de Barra $M$, Aunger R. Disgust as an adaptive system for disease avoidance behaviour. Philos Trans R Soc Lond B Biol Sci. 2011;366:389401.

33. da Silva AG, Miranda DM, Diaz AP, Teles AL, MalloyDiniz LF, Palha AP. Mental health: why it still matters in the midst of a pandemic. Braz J Psychiatry. 2020 Apr 3;S1516-44462020005008202. doi: 10.1590/1516-4446-2020-0009. Online ahead of print.

34. Duan L, Zhu G. Psychological interventions for people affected by the COVID-19 epidemic. Lancet Psychiatry 2020;7:300-2.

35. Correa H, Malloy-Diniz LF, da Silva AG. Why psychiatric treatment must not be neglected during the COVID-19 pandemic. Braz J Psychiatry. 2020 Apr 30;S1516-44462020005011204. doi: 10.1590/1516-4446-2020-0995. Online ahead of print.

36. Yao $H$, Chen JH, Xu YF. Patients with mental health disorders in the COVID-19 epidemic. Lancet Psychiatry. 2020;7:e21. 